\title{
FRONTIERS IN COMPLEX DYNAMICS
}

\author{
CURTIS T. MCMULLEN
}

\section{INTRODUCTION}

Rational maps on the Riemann sphere occupy a distinguished niche in the general theory of smooth dynamical systems. First, rational maps are complexanalytic, so a broad spectrum of techniques can contribute to their study (quasiconformal mappings, potential theory, algebraic geometry, etc.). The rational maps of a given degree form a finite-dimensional manifold, so exploration of this parameter space is especially tractable. Finally, some of the conjectures once proposed for smooth dynamical systems (and now known to be false) seem to have a definite chance of holding in the arena of rational maps.

In this article we survey a small constellation of such conjectures centering around the density of hyperbolic rational maps - those which are dynamically the best behaved. We discuss some of the evidence and logic underlying these conjectures, and sketch recent progress towards their resolution.

Our presentation entails only a brief account of the basics of complex dynamics; a more systematic exposition can be found in the survey articles [Dou1], $[\mathrm{BI}]$, and [EL]; the recent books [Bea] and [CG]; and Milnor's lecture notes [Mil2].

\section{HyPERBolic RATIONAL MAPS}

A rational map $f: \widehat{\mathbb{C}} \rightarrow \widehat{\mathbb{C}}$ is a holomorphic dynamical system on the Riemann sphere $\widehat{\mathbb{C}}=\mathbb{C} \cup\{\infty\}$. Any such map can be written as a quotient

$$
f(z)=\frac{P(z)}{Q(z)}=\frac{a_{0} z^{d}+\ldots+a_{d}}{b_{0} z^{d}+\ldots+b_{d}}
$$

of two relative prime polynomials $P$ and $Q$. The degree of $f$ can be defined topologically or algebraically; it is the number of preimages of a typical point $z$, as well as the maximum of the degrees of $P$ and $Q$. The fundamental problem in the dynamics of rational maps is to understand the behavior of high iterates

$$
f^{n}(z)=\underbrace{(f \circ f \circ \ldots \circ f)}_{n \text { times }}(z) .
$$

Any rational map of degree $d>1$ has both expanding and contracting features. For example, $f$ must be expanding on average, because it maps the

Received by the editors February 1, 1994.

1991 Mathematics Subject Classification. Primary 30D05, 58F23.

Based on a lecture presented to the AMS-CMS-MAA joint meeting, Vancouver, BC, August 16, 1993. Supported in part by the NSF. 
Riemann sphere over itself $d$ times. Indeed, with respect to the spherical metric (normalized to have total area one),

$$
\int_{\widehat{\mathbb{C}}}\left\|\left(f^{n}\right)^{\prime}\right\|^{2}=d^{n} \rightarrow \infty
$$

so the derivative of $f^{n}$ is very large on average. On the other hand, $f$ has $2 d-2$ critical points $c$ where $f^{\prime}(c)=0$. Near $c$, the behavior of $f$ is like that of $z \mapsto z^{n}$ near the origin, for some $n>1$; thus $f$ is highly contracting near $c$. Tension between these two aspects of $f$ is responsible for much of the complexity of rational maps.

To organize these features of $f$, we introduce the Julia set $J(f)$ - the locus of chaotic dynamics; and the postcritical set $P(f)$ - which contains the "attractors" of $f$. The Julia set can be defined as the closure of the set of repelling periodic points for $f$. Here a point $z$ is periodic if $f^{p}(z)=z$ for some $p>0$; it is

$$
\begin{array}{ll}
\text { repelling } & \text { if }\left|\left(f^{p}\right)^{\prime}(z)\right|>1, \\
\text { indifferent } & \text { if }\left|\left(f^{p}\right)^{\prime}(z)\right|=1, \text { and } \\
\text { attracting } & \text { if }\left|\left(f^{p}\right)^{\prime}(z)\right|<1 .
\end{array}
$$

The forward orbit $E$ of a periodic point is called a cycle, because $f \mid E$ is a cyclic permutation.

The derivative gives a first approximation to the behavior of $f^{p}$ near the periodic point; for example, all points in a small neighborhood of an attracting point $z$ tend towards $z$ under iteration of $f^{p}$. On the other hand, a repelling point pushes away nearby points, so the behavior of forward iterates is difficult to predict.

The Julia set is also the smallest closed subset of the sphere such that $|J(f)|>$ 2 and $f^{-1}(J)=J$. Its complement, $\Omega=\widehat{\mathbb{C}}-J(f)$, sometimes called the Fatou set, is the largest open set such that the iterates $\left\langle f^{n} \mid \Omega\right\rangle$ form a normal family.

The postcritical set $P(f)$ is the closure of the forward orbits of the critical points of $f$ :

$$
P(f)=\overline{\bigcup_{n>0, f^{\prime}(c)=0} f^{n}(c)}
$$

The postcritical set plays a crucial role with respect to the attractors of $f$. For example:

Theorem 2.1. Every attracting cycle $A$ attracts a critical point.

Proof. Let $U=\left\{z: d\left(f^{n}(z), A\right) \rightarrow 0\right\}$ for the spherical metric; $U$ is open, and $f^{-1}(U)=U$. If $U$ contains no critical point, then $f \mid U$ is a covering map; but then the Schwarz lemma implies $f$ is an isometry for the hyperbolic metric, which is impossible because $A$ is attracting.

Thus $A \subset P(f)$ and the number of attracting cycles is bounded by the number of critical points, which in turn is bounded by $2 \operatorname{deg}(f)-2$.

This theorem is of practical as well as theoretical value. For example, if $f(z)=z^{2}+c$ has an attracting cycle of period 100 , this cycle can be easily located as $\lim f^{n}(0)$; a few million iterates should yield reasonable accuracy. (Compare this to the prospect of computing the $1.27 \times 10^{30}$ roots of the equation $f^{100}(z)=z$.)

We can now introduce the property of hyperbolicity, which will be central in the remaining discussion. Let $f$ be a rational map of degree $d>1$. 
Theorem 2.2. The following conditions are equivalent:

(1) All critical points of $f$ tend to attracting cycles under iteration.

(2) The map $f$ is expanding on its Julia set. That is, there exists a conformal metric $\rho$ on the sphere such that $\left|f^{\prime}(z)\right|_{\rho}>1$ for all $z \in J(f)$.

(3) The postcritical set and the Julia set are disjoint $(P(f) \cap J(f)=\emptyset)$.

Definition. When the above conditions hold, $f$ is hyperbolic.

The Julia set of a hyperbolic rational map is thin: its area is zero, and in fact its Hausdorff dimension is strictly less than two [Sul2]. Every point outside the Julia set tends towards a finite attractor $A \subset \widehat{\mathbb{C}}$ : that is, the spherical distance $d\left(f^{n}(z), A\right) \rightarrow 0$ as $n \rightarrow \infty$. The set $A$ consists exactly of the union of the attracting cycles for $f$.

Thus for a hyperbolic rational map, we can predict the asymptotic behavior of all points in an open, full-measure subset of the sphere: they converge to $A$.

Example. Figure 1 depicts the Julia set of a rational map of degree 11. The Julia set is in black; its complement contains twenty large white regions, ten of which are visible in the picture. The attractor $A$ consists of one point in the "center" of each large white region. Under iteration, every point outside the Julia set eventually lands in one of the large white regions and is then attracted to its center. The Julia set is the thin set of "indecisive" points forming the boundary between regions converging to one point of $A$ or to another.

This rational map is especially symmetric: it commutes with the symmetries of the dodecahedron, and it can be used to solve the quintic equation (but that is another story; see [DMc]).

We can now state one of the central open problems in the field.

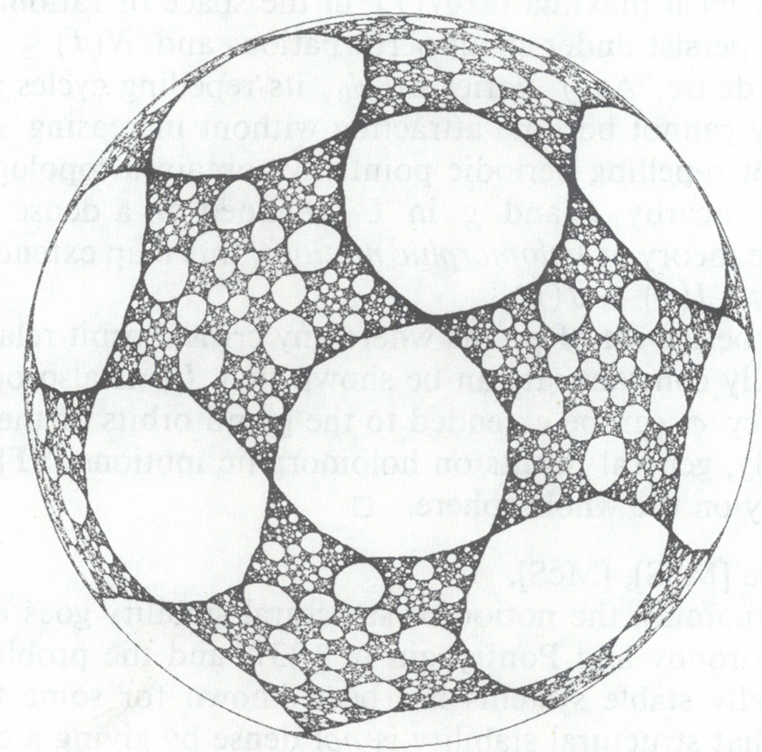

FIGURE 1. Julia set of a hyperbolic rational map with the symmetries of the icoashedron. 
Conjecture HD. Hyperbolic maps are open and dense among all rational maps.

It is easy to see that hyperbolicity is an open condition, but the density of hyperbolic dynamics has so far eluded proof.

Given recent events in number theory, I looked into the possibility of naming the above conjecture Fatou's Last Theorem. Unfortunately, the name is unjustified. Speaking of hyperbolicity, Fatou writes in his 1919-20 memoir [Fatou, p.73]:

Il est probable, mais je n'ai pas approfondi la question, que cette propriété appartient à toutes les substitutions générales, c'està-dire celles dont les coefficients ne vérifient aucune relation particulière. $^{1}$

There is no indication of even a marginal proof. Moreover, Fatou may have intended by his last statement that the non-hyperbolic rational maps should be contained in a countable union of proper subvarieties. This is false, by an elementary argument [Lyu 1, Proposition 3.4]; in fact, non-hyperbolic maps have positive measure among all rational maps of a given degree [Rees1].

Structural stability. A pair of rational maps $f$ and $g$ are topologically conjugate if there is a homeomorphism $\phi: \widehat{\mathbb{C}} \rightarrow \widehat{\mathbb{C}}$ such that $\phi f \phi^{-1}=g$. A rational map $f$ is structurally stable if $f$ is topologically conjugate to all $g$ in a neighborhood of $f$.

The following close relative of Conjecture HD is known to be true:

Theorem 2.3 (Mañé, Sad, Sullivan). The set of structurally stable rational maps is open and dense.

Sketch of the proof. Let $N(f)$ be the number of attracting cycles of $f$, and let $U_{0}$ be the set of local maxima of $N(f)$ in the space of rational maps. Since attracting cycles persist under small perturbations and $N(f) \leq 2 d-2$, the set $U_{0}$ is open and dense. As $f$ varies in $U_{0}$, its repelling cycles are persistently repelling - they cannot become attracting without increasing $N(f)$. Tracing the movement of repelling periodic points, we obtain a topological conjugacy between any two nearby $f$ and $g$ in $U_{0}$, defined on a dense subset of their Julia sets. By the theory of holomorphic motions, this map extends continuously to a conjugacy $\phi: J(f) \rightarrow J(g)$.

Let $U_{1} \subset U_{0}$ be the set of points where any critical orbit relations $\left(f^{n}(c)=\right.$ $\left.f^{m}\left(c^{\prime}\right)\right)$ are locally constant. It can be shown that $U_{1}$ is also open and dense, and the conjugacy $\phi$ can be extended to the grand orbits of the critical points over $U_{1}$. Finally, general results on holomorphic motions [ST], [BR] prolong $\phi$ to a conjugacy on the whole sphere.

For details see [MSS], [McS].

In smooth dynamics, the notion of structural stability goes back at least to the work of Andronov and Pontryagin in 1937, and the problem of the density of structurally stable systems has been known for some time. In 1965 Smale showed that structural stability is not dense by giving a counterexample in the space of diffeomorphisms on a 3-torus [Smale]. Eventually it was found

\footnotetext{
${ }^{1}$ I am grateful to Eremenko, Lyubich, and Milnor for providing this reference.
} 
that neither structural stability nor hyperbolicity is dense in the space of diffeomorphisms, even on 2-dimensional manifolds (see articles by Abrahams-Smale, Newhouse, Smale, and Williams in [CS]).

It is thus remarkable that structural stability is dense within the space of rational maps; this fact highlights the special character of these more rigid dynamical systems. Given the density of structural stability, to settle Conjecture HD it suffices to prove that a structurally stable rational map is hyperbolic.

More recent results in smooth dynamics actually support Conjecture HD; the implication (structural stability) $\Longrightarrow$ (hyperbolicity) is now known to hold for $C^{1}$ diffeomorphisms [Mane].

\section{INVARIANT LINE FIELDS}

What further evidence can be offered for Conjecture HD?

Theoretical support is provided by a more fundamental conjecture, which has its roots in the quasiconformal deformation theory of rational maps and relates to Mostow rigidity of hyperbolic 3-manifolds. To describe this conjecture, we will first give an example of a non-hyperbolic rational map - indeed, a rational map whose Julia set is the entire Riemann sphere.

The construction begins with a complex torus $X=\mathbb{C} / \Lambda$, where $\Lambda=\mathbb{Z} \oplus \tau \mathbb{Z}$ is a lattice in the complex plane. Choose $n>1$, and let $F: X \rightarrow X$ be the degree $n^{2}$ holomorphic endomorphism given by $F(x)=n x$. Since $\left|F^{\prime}(x)\right|=$ $n>1$, the map $F$ is uniformly expanding, and it is easy to see that repelling periodic points of $F$ are dense on the torus $X$. (For example, all points of order $n^{k}-1$ in the group law on $X$ have period $k$ under $F$.) Thus the Julia set of $F$, appropriately interpreted, is the whole of $X$.

The quotient of $X$ by the equivalence relation $x \sim-x$ is the Riemann sphere; the quotient map $\wp: X \rightarrow \widehat{\mathbb{C}}$ can be given by the Weierstrass $\wp$ function, which presents $X$ as a twofold cover of the sphere branched over four points. Since $F(-x)=-F(x)$, the dynamical system $F$ descends to a rational map $f$ such that the diagram

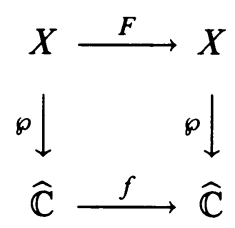

commutes.

The mapping $f$ can be thought of as an analogue of the multiple angle formulas for sine and cosine, since $f(\wp(z))=\wp(n z)$.

Definition. A rational map $f$ is covered by an integral torus endomorphism if it arises by the construction above. ${ }^{2}$

Here are some remarkable features of $f$ :

(1) The Julia set $J(f)=\widehat{\mathbb{C}}$. This follows easily from the density of repelling periodic points for $F$ on $X$.

\footnotetext{
${ }^{2}$ This construction goes back to Lattès [Lat].
} 
(2) The mapping $f$ is not rigid: that is, by deforming the lattice $\Lambda$ (varying $\tau$ ), we obtain a family of rational maps which are topologically conjugate but not conformally conjugate.

(3) Most importantly, the Julia set $J(f)$ carries an invariant line field. To visualize this line field, first note that the map $z \mapsto n z$ preserves the family of horizontal lines in the complex plane. Thus $F$ preserves the images of such lines on the torus. The quotient line family turns out to be a foliation by parallel simple closed geodesics (with respect to the obvious Euclidean metric) on the torus. Finally, $f$ preserves the image of this foliation on the sphere.

Of course there is no way to comb a sphere, so the image foliation has singularities: there are four singular points at the four critical values of $\wp$.

More generally, an invariant line field for $f$, defined on a measurable set $E \subset \widehat{\mathbb{C}}$, is the choice of a one-dimensional real subspace $L_{z}$ in the tangent space $T_{z} \widehat{\mathbb{C}}$ for all $z \in E$, such that:

(1) $E$ has positive area,

(2) $f^{-1}(E)=E$,

(3) the slope of $L_{z}$ varies measurably with respect to $z$, and

(4) the derivative $f^{\prime}$ transforms $L_{z}$ into $L_{f(z)}$ for all $z$ in $E$.

If $E \subset J(f)$, we say $f$ admits an invariant line field on its Julia set. Thus the Julia set must have positive measure before it can carry an invariant line field.

Conjecture NILF. A rational map $f$ carries no invariant line field on its Julia set, except when $f$ is covered by an integral torus endomorphism.

This conjecture is stronger than the density of hyperbolic dynamics:

Theorem 3.1 (Mañé, Sad, Sullivan). NILF $\Longrightarrow H D$.

See [MSS], [McS].

One attractive feature of conjecture NILF is that it shifts the focus of study from the family of all rational maps to the ergodic theory of a single rational map.

In support of this conjecture, and hence of the density of hyperbolic dynamics, we state a parallel result for degree one rational maps. Of course a single degree one rational map is not very complicated. The degree one mappings form a group, isomorphic to $\mathrm{PSL}_{2}(\mathbb{C})$, and the group structure makes it easy to iterate a single mapping. To make the dynamical system more interesting, let us consider more generally finitely generated subgroups $\Gamma \subset \mathrm{PSL}_{2}(\mathbb{C})$ and define the Julia set $J(\Gamma)$ as the minimal closed invariant set with $|J|>2$. We then have:

Theorem 3.2 (Sullivan). A discrete finitely generated group $\Gamma$ of degree one rational maps carries no invariant line field on its Julia set.

This result is a (thinly disguised) version of Mostow rigidity for hyperbolic 3-manifolds and orbifolds with finitely generated fundamental group [Sul1]; in more traditional terminology, $\Gamma$ is a Kleinian group and $J(\Gamma)$ is its limit set.

If we allow $\Gamma$ to be an indiscrete subgroup of $\mathrm{PSL}_{2}(\mathbb{C})$, the theorem fails, but in a completely understood way. For example, the group

$$
\Gamma=\langle z \mapsto z+1, z \mapsto z+\tau, z \mapsto n z\rangle,
$$


with $\operatorname{Im}(\tau)>0$ and $n>1$, has $J(\Gamma)=\widehat{\mathbb{C}}$, and it preserves the field of horizontal lines in $\mathbb{C}$. This example is simply the universal cover of a torus endomorphism; in a sense, the exceptions proposed in conjecture NILF correspond to the (easily classified) case of indiscrete groups.

With this result to guide us, why has the no-invariant-line-field conjecture remained elusive? The main reason is perhaps that all rational maps of degree one lie in a finite-dimensional Lie group. This group provides a good geometric portrait of an arbitrary degree one transformation. By contrast, the degree of a general rational map can tend to infinity under iteration, and it is much more difficult to visualize and control the behavior of a rational map of high degree.

\section{QUADRATIC POLYNOMIALS}

The simplest rational maps, apart from those of degree one, are the quadratic polynomials. To try to gain insight into the general theory of rational maps, much effort has been devoted to this special case. The quadratic polynomials are remarkably rich in structure, and many fundamental difficulties are already present in this family.

From the point of view of dynamics, every quadratic polynomial occurs exactly once in the family

$$
f_{c}(z)=z^{2}+c \quad(c \in \mathbb{C}),
$$

so the quadratic parameter space can be identified with the complex plane.

Restricting attention from rational maps to quadratic polynomials, it is natural to formulate the following conjectures.

Conjecture HD2. Hyperbolic maps are dense among quadratic polynomials.

Conjecture NILF2. A quadratic polynomial admits no invariant line field on its Julia set.

It turns out that these two conjectures are equivalent. This equivalence is further evidence for the fundamental nature of the question of invariant line fields.

Note that $f_{c}$ has only one critical point in the complex plane, namely, $z=0$. Consequently:

Theorem 4.1. The map $f_{c}(z)=z^{2}+c$ is hyperbolic if and only if $f_{c}^{n}(0) \rightarrow \infty$ or $f_{c}$ has an attracting periodic cycle in the finite plane $\mathbb{C}$.

This theorem motivates the following:

Definition. The Mandelbrot set $M \subset \mathbb{C}$ is the set of $c$ such that $f_{c}^{n}(0)$ stays bounded as $n \rightarrow \infty$.

The Mandelbrot set is compact, connected, and full (this means $\mathbb{C}-M$ is also connected). The interior of $M$ consists of countably many components, appearing as bounded white regions in Figure 2. Thus $M$ can be thought of as a "tree with fruit", the fruit being the components of its interior (cf. [Dou2]).

Where does the fruit come from?

Conjecture $\mathrm{HD2}^{\prime}$. If $c$ lies in the interior of the Mandelbrot set, then $f_{c}(z)$ has an attracting cycle. 


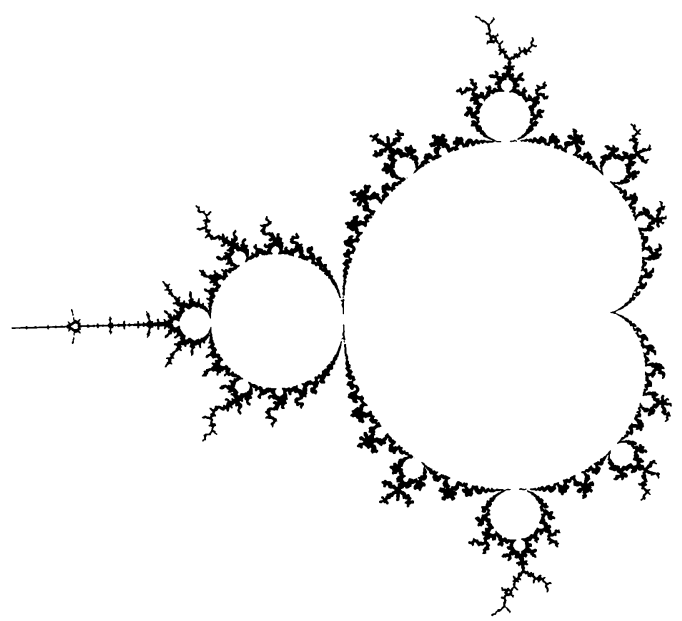

FIGURE 2. The boundary of the Mandelbrot set.

It turns out that hyperbolicity is infectious - if $U$ is a component of the interior of the Mandelbrot set, and $f_{c}$ is hyperbolic for one $c \in U$, then $f_{c}$ is hyperbolic for all $c \in U$. In this case we say $U$ is a hyperbolic component of $\operatorname{int}(M)$.

It follows that Conjecture HD2' is also equivalent to Conjecture HD2, so several natural conjectures concur in the setting of complex quadratic polynomials.

Real quadratic polynomials. The Mandelbrot set meets in the real axis (the horizontal line of symmetry in Figure 2) in the interval $[-2,1 / 4]$. We can further specialize the conjectures above to real quadratics, obtaining:

Conjecture HD2 $\mathbb{R}$. Hyperbolicity is dense among real quadratic polynomials.

Conjecture NILF2 $\mathbb{R}$. A real quadratic polynomial admits no invariant line field on its Julia set.

The real quadratic polynomials are of special interest for several reasons. First, there are many dynamical systems which can be roughly modeled on such a polynomial: the economy, animal populations, college enrollment, etc. To explain this, it is convenient to conjugate a real polynomial in $M$ to the form $g(x)=\lambda x(1-x)$, where $0<\lambda \leq 4$, so $g:[0,1] \rightarrow[0,1]$. Then $g$ is a "unimodal map": for $x>0$ small $g(x)$ grows as $x$ grows; but after $x$ passes a critical point $(x=1 / 2), g$ decreases as $x$ increases. Thus $g$ might describe the boom and bust of economic cycles or the behavior of population from one year to the next when faced with limited resources. See Figure 3, which plots $g$ together with the diagonal $y=x$ and shows an example where the critical point has period 8 .

One can imagine that real numbers correspond to real life, and one goal of the complex theory is ultimately to contribute to the understanding of dynamics over the reals.

Second, some of the combinatorial and geometric analysis of a quadratic polynomial becomes especially tractable over the real numbers, because of the order structure on the real line. For example, the forward orbit $\left\langle f_{c}^{n}(0)\right\rangle$ of the 


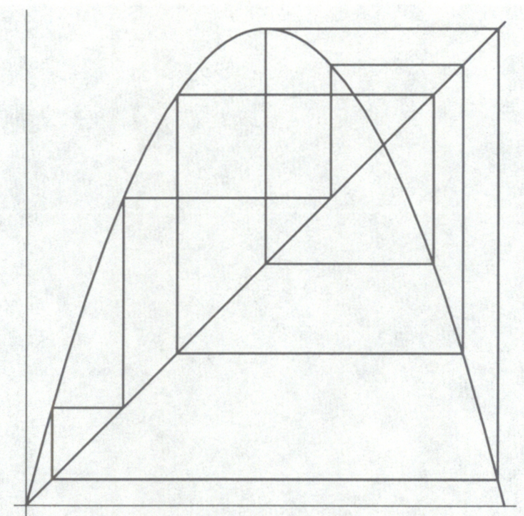

FIGURE 3. Real quadratic with periodic critical point.

critical point is real when $c \in \mathbb{R}$, so the postcritical set $P\left(f_{c}\right)$ is thin and cannot double back on itself.

Finally, if we consider $z^{2}+c$ with both $z$ and $c$ real, we can conveniently draw two-dimensional pictures displaying dynamical features on the $z$ line as the parameter $c$ varies.

One such classic computer experiment is the following. For $c \in \mathbb{R}$, let

$$
A_{c}=\left\{\text { limit points of } f_{c}^{n}(0) \text { as } n \rightarrow \infty\right\} \subset \mathbb{R}
$$

denote the "attractor" of $f_{c}$. If $f_{c}$ has an attracting cycle, then $A_{c}$ will be equal to that finite set. On the other hand, if $A_{c}$ is an infinite, then $f_{c}$ cannot be hyperbolic.

Now draw the set $\left\{(x, c): x \in A_{c}\right\}$ as $c$ varies along the real axis in the Mandelbrot set in the negative direction, starting just within the main cardioid; the result appears in Figure 4. In the main cardioid of $M, f_{c}$ has an attracting

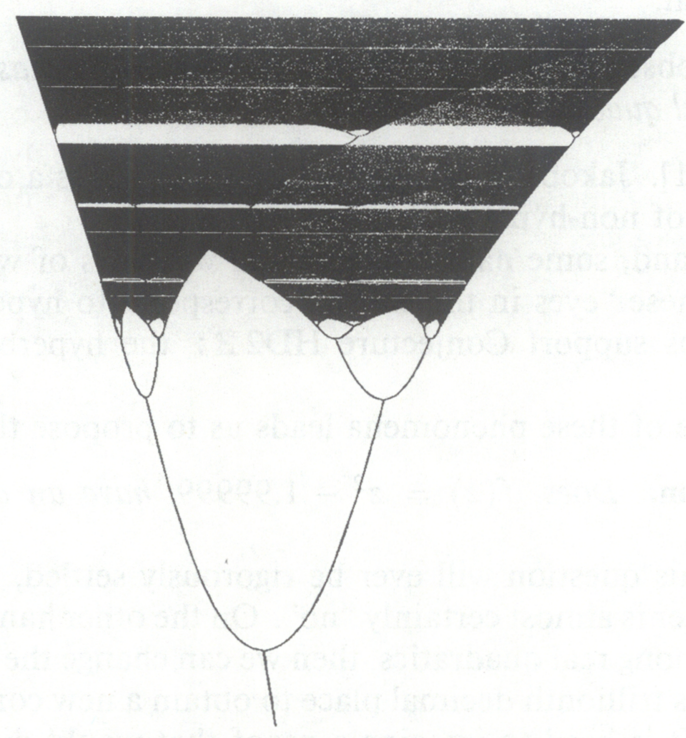

FigURE 4. Bifurcation diagram. 


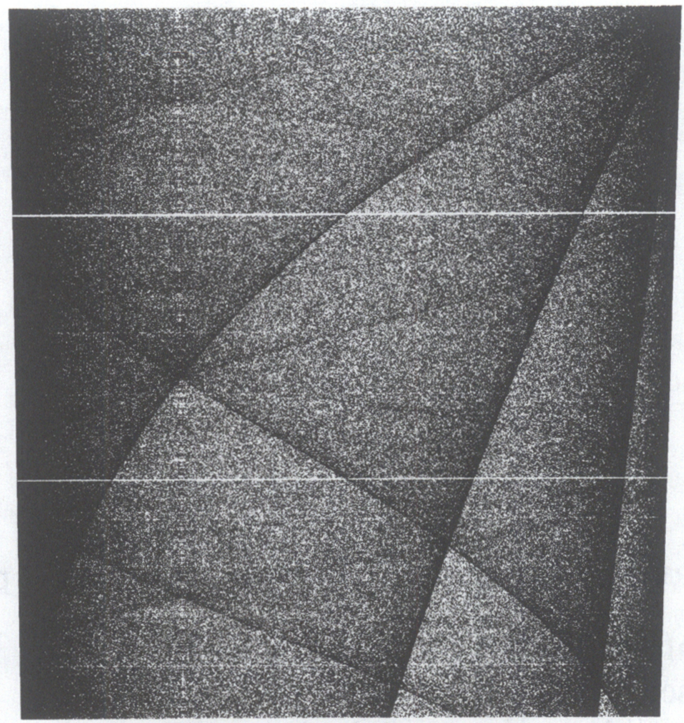

Figure 5. Blowup near $c=-2$.

fixed point, so at the bottom of the figure $A_{c}$ consists of a single point. As $c$ decreases, this point bifurcates to a cycle of period 2, which in turn bifurcates to period 4, 8, and so on. Above this "cascade of period doublings" the structure becomes very complicated and the picture is much darker; there are large sets of $c$ such that $A_{c}$ is an entire interval and the corresponding map is far from hyperbolic. The top of the figure corresponds to $c=-2$.

A blowup of the region near $c=-2$ appears in Figure 5. (The prominent smooth curves in this picture come from the forward orbit of the critical point.) This picture makes apparent the ubiquity of chaotic dynamics. In fact, we have the following result:

Theorem 4.2 (Jakobson). The set of non-hyperbolic maps has positive measure in the space of real quadratic polynomials.

See [Jak], [Yoc1]. Jakobson also shows that $c=-2$ is a one-sided point of density of the set of non-hyperbolic maps.

On the other hand, some narrow horizontal windows of white are also visible in Figure 5; these "eyes in the storm" correspond to hyperbolic maps, and successive blowups support Conjecture HD2 $\mathbb{R}$ : the hyperbolic windows are apparently dense.

The coexistence of these phenomena leads us to propose the following:

Challenge Question. Does $f(z)=z^{2}-1.99999$ have an attracting periodic point?

It is unlikely this question will ever be rigorously settled, for by Jakobson's theorem, the answer is almost certainly "no". On the other hand, if hyperbolicity is indeed dense among real quadratics, then we can change the constant 1.99999 somewhere past its trillionth decimal place to obtain a new conjecture where the answer is "yes". It is hard to imagine a proof that would distinguish between these two cases. 
We can sum up the conjectures put forth so far, and known implications between them, in the following table.

\begin{tabular}{|c|c|c|}
\hline & $\begin{array}{c}\text { Hyperbolic } \\
\text { maps are dense }\end{array}$ & $\begin{array}{l}\text { No invariant } \\
\text { line fields }\end{array}$ \\
\hline Rational maps & $\mathrm{HD} \Longleftarrow$ & NILF \\
\hline Quadratic polynomials & $\mathrm{HD} 2 \Longleftrightarrow$ & $\underset{\Downarrow}{\text { NILF2 }}$ \\
\hline Real quadratic polynomials & $\mathrm{HD} 2 \mathbb{R} \Longrightarrow$ & NILF2 $\mathbb{R}$ \\
\hline
\end{tabular}

Remarkably, the fundamental conjectures concerning quadratic polynomials (real or complex) can be subsumed into the following topological statement:

Conjecture MLC. The Mandelbrot set is locally connected.

Theorem 4.3 (Douady-Hubbard). $M L C \Longrightarrow H D 2, H D 2 \mathbb{R}, N I L F 2$, and $N I L F 2 \mathbb{R}$.

Why is locally connectivity such a powerful property? One answer comes from a theorem of Carathéodory, which states that the Riemann mapping

$$
\psi:(\mathbb{C}-\bar{\Delta}) \rightarrow(\mathbb{C}-M)
$$

extends to a continuous map $S^{1} \rightarrow \partial M$ if and only if $\partial M$ is locally connected. (Here $\Delta$ is the unit disk and $S^{1}=\partial \Delta$. The Riemann mapping is normalized so that $\psi(z) / z \rightarrow 1$ as $z \rightarrow \infty$.)

If $M$ is locally connected, then each point $\exp (2 \pi i t) \in S^{1}$ corresponds to a unique point $c$ in $\partial M$. The external angle $t$ is a sort of generalized rotation number, and indeed the mappings corresponding to rational values of $t$ are well understood. On the other hand, the combinatorics of $f_{c}$ determines the (one or more) external angles $t$ to which it corresponds.

If $M$ is locally connected, then a quadratic polynomial $f_{c}$ with $c \in \partial M$ is determined by its combinatorics, even for irrational external angles. Using this information, one can build an abstract model for $M$ which is topologically correct; since the density of hyperbolicity is a topological notion, it suffices to check it in the abstract model, and Conjectures HD2 and HD2 $\mathbb{R}$ follow.

\section{RENORMALIZATION}

We next present some recent breakthroughs in the direction of the conjectures above. To explain these results, we will need the concept of renormalization.

The local behavior of a rational map can sometimes be given a linear model. For example, near a repelling fixed point $p$ with $f^{\prime}(p)=\lambda$, one can choose a complex coordinate $z$ so that the dynamics take the form $f: z \mapsto \lambda z$.

Renormalization is simply nonlinear linearization; that is, one looks for a local model of the dynamics which is a polynomial of degree greater than one. We will make this precise in the context of quadratic polynomials.

Let $f(z)=z^{2}+c$ with $c$ in the Mandelbrot set. An iterate $f^{n}$ is renormalizable if there exist disks $U$ and $V$ containing the origin, with $\bar{U}$ a compact subset of $V$, such that (a) $f^{n}: U \rightarrow V$ is a proper map of degree two and (b) $f^{n k}(0) \in U$ for all $k>0$. This means that although $f^{n}$ is a polynomial 


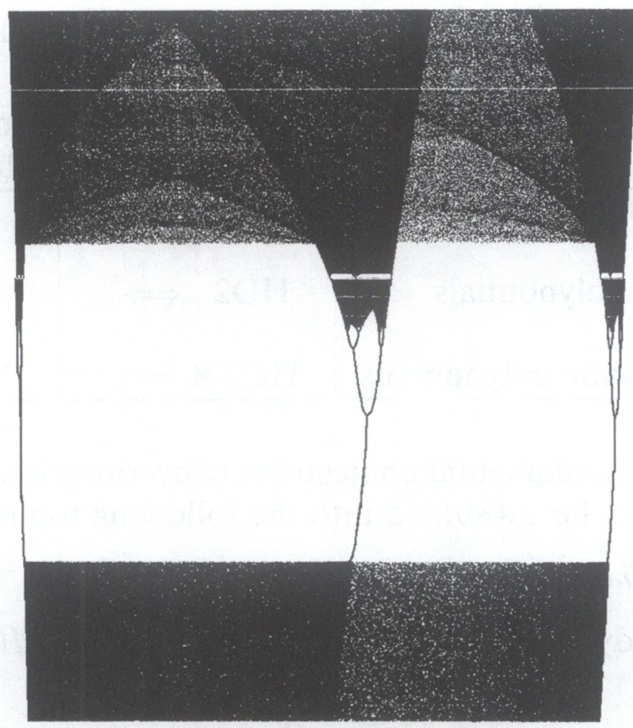

FIGURE 6. Recapitulation of bifurcation.

of degree $2^{n}$, it behaves like a polynomial of degree two on a suitable neighborhood of the critical point $z=0$. The restriction $f^{n}: U \rightarrow V$ is called a quadratic-like map. A fundamental theorem of Douady and Hubbard asserts that any quadratic-like map is topologically conjugate to a quadratic polynomial $g(z)=z^{2}+c^{\prime}$; condition (b) implies $c^{\prime}$ lies in the Mandelbrot set and, with suitable normalizations, is unique [DH].

The concept of renormalization explains much of the self-similarity in the Mandelbrot set and in the bifurcation diagram for real quadratic polynomials. For example, there is a prominent window of white in the midst of the chaotic regime of Figure 4; a blow-up of this region appears in Figure 6. Remarkably, three small copies of the entire bifurcation diagram appear. This is explained by the fact that $f^{3}$ is renormalizable for all values of $c$ in this window. As $c$ traverses the window, the quadratic-like maps $f_{c}^{3}: U_{c} \rightarrow V_{c}$ recapitulate the full family of bifurcations of a quadratic polynomial. (In the Mandelbrot set, one finds a small homeomorphic copy of $M$ framing this window on the real axis.)

Infinite renormalization. A quadratic polynomial $f$ is infinitely renormalizable if $f^{n}$ is renormalizable for infinitely many $n>1$.

The prime example of an infinitely renormalizable mapping is the Feigenbaum polynomial $f(z)=z^{2}-1.401155 \ldots$. For this map, a suitable restriction of $f^{2}$ is a quadratic-like map topologically conjugate to $f$ itself. It follows that $f^{2^{n}}$ is renormalizable for every $n \geq 1$. Its attractor $A_{c}$ is a Cantor set representing the limit of the cascade of period doublings visible in Figure 4. This Cantor set, the map $f$, and the cascade of period doublings all exhibit remarkable universal scaling features that physicists associate with phase transitions and that have studied for many years (see, e.g. the collection [Cvi]).

Techniques from complex analysis and Teichmüller theory have been brought to bear by Sullivan to provide a conceptual understanding of this universality 
[Sul3]. At the moment the theory applies only to real quadratics, that is, $z^{2}+c$ with $c \in \mathbb{R}$; however, there is little doubt that universality exists over $\mathbb{C}$ [Mil1].

Infinitely renormalizable mappings are very special. Remarkably, great progress has been made towards understanding all other quadratic polynomials and settling for them the conjectures discussed in this paper. The central result is:

Theorem 5.1 (Yoccoz). If $c$ belongs to the Mandelbrot set, then either:

$f_{c}(z)=z^{2}+c$ is infinitely renormalizable, or

$J\left(f_{c}\right)$ admits no invariant line field and $M$ is locally connected at $c$.

Yoccoz's theorem was anticipated by a breakthrough in cubic polynomials due to Branner and Hubbard [BH2], and we will use their language of tableaux to describe Yoccoz's proof. (See also [Mil3], [Hub], and [Yoc2].)

Sketch of the proof. Suppose $c \in M$. Let $K\left(f_{c}\right)$ denote the filled Julia set, that is, the set of $z \in \mathbb{C}$ which remains bounded under iteration of $f_{c}$; its boundary is the Julia set, and $K\left(f_{c}\right)$ is connected.

Let

$$
\phi_{c}:(\mathbb{C}-\bar{\Delta}) \rightarrow\left(\mathbb{C}-K\left(f_{c}\right)\right)
$$

be the Riemann mapping, normalized so that $\phi_{c}^{\prime}(z)=1$ at infinity. It is easy to see that

$$
\phi_{c}\left(z^{2}\right)=f_{c}\left(\phi_{c}(z)\right)
$$

in other words, $\phi_{c}$ conjugates the $z^{2}$ to $f_{c}$.

An external ray $R_{t}$ is the image of the ray $(1, \infty) \exp (2 \pi i t)$ under the mapping $\phi_{c}$; similarly, an external circle $C_{r}$ (also called an equipotential) is the image of $\{z:|z|=r\}$. Note that $f_{c}\left(R_{t}\right)=R_{2 t}$ and $f_{c}\left(C_{r}\right)=C_{r^{2}}$ by the functional equation for $\phi_{c}$.

The main case of the proof arises when all periodic cycles of $f_{c}$ are repelling; let us assume this. The first step is to try to show that the Julia set $J\left(f_{c}\right)$ is locally connected. To this end, Yoccoz constructs a sequence $\left\langle\mathscr{P}_{d}\right\rangle$ of successively finer tilings of neighborhoods of $J\left(f_{c}\right)$.

To illustrate the method, consider the special case $c=i$. For this map, the external rays $R_{1 / 7}, R_{2 / 7}$, and $R_{4 / 7}$ converge to a repelling fixed point $\alpha$ of $f_{c}$. These rays cut the disk bounded by the external circle $C_{2}$ into three tiles (see Figure 7) called the puzzle pieces $\mathscr{P}_{0}$ at level 0 . The pieces at level $d+1$ are defined inductively as the components of the preimages of the pieces $\mathscr{P}_{d}$ at level $d$. The new pieces fit neatly inside those already defined, because the external rays converging to $\alpha$ are forward-invariant.

The puzzle pieces provide connected neighborhoods of points in the Julia set. To show $\boldsymbol{J}\left(f_{c}\right)$ is locally connected, it suffices to show that $\operatorname{diam}\left(\boldsymbol{P}_{d}\right) \rightarrow 0$ for any nested sequence of pieces $P_{0} \supset P_{1} \supset P_{2} \supset \ldots$.

Now $\operatorname{diam}\left(P_{i}\right) \rightarrow 0$ will follow if we can establish

$$
\sum \bmod \left(P_{i}-P_{i+1}\right)=\infty ;
$$

here each region $P_{i}-P_{i+1}$ is a (possibly degenerate) annulus, and the modulus $\bmod (A)=m$ if the annulus $A$ is conformally isomorphic to the standard round annulus $\{z: 1<|z|<\exp (2 \pi m)\}$. 


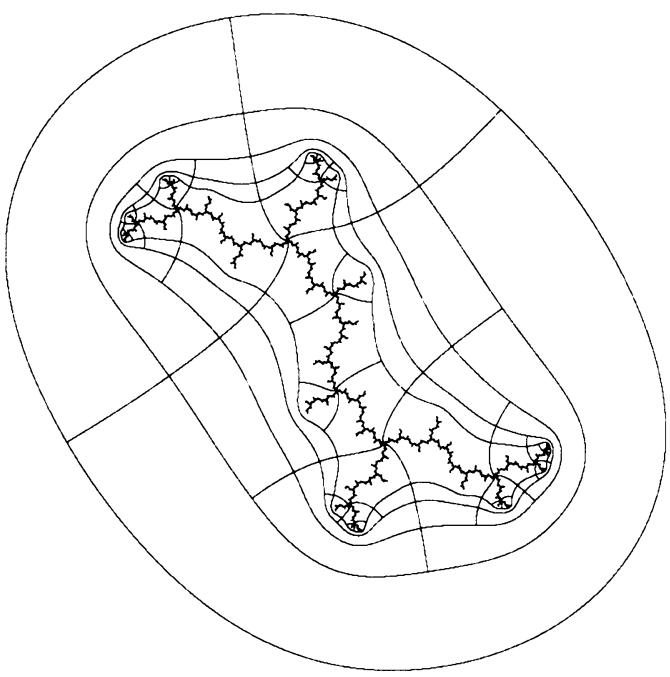

Figure 7. The Yoccoz puzzle.

The modulus is especially useful in holomorphic dynamics because it is invariant under conformal mappings; more generally, $\bmod \left(A^{\prime}\right)=\bmod (A) / d$ if $A^{\prime}$ is a $d$-fold covering of an annulus $A$.

Since the image of a puzzle piece of depth $d>0$ under $f_{c}$ is again a puzzle piece, the moduli of the various annuli that can be formed satisfy many relations. Roughly speaking, the tableau method allows one to organize these relations and test for divergence of sums of moduli.

For degree two polynomials, the method succeeds unless certain annuli are repeatedly covered by degree two. Unfortunately, this exceptional case leads to the convergent sum $1+1 / 2+1 / 4+\ldots$, and so it does not prove local connectivity. However, one finds this case only occurs when the polynomial is renormalizable.

The case of a finitely renormalizable map $f_{c}$ can be handled by respecifying the initial tiling $\mathscr{P}_{0}$. Thus the method establishes locally connectivity of $J\left(f_{c}\right)$ unless the mapping is infinitely renormalizable.

It is a metatheorem that the structure of the Mandelbrot set at $c$ reflects properties of the Julia set $J\left(f_{c}\right)$. In this case the proof of locally connectivity of $J\left(f_{c}\right)$ can be adapted, with some difficulty, to establish locally connectivity of $M$ at $c$. A variant of Theorem 4.3 then shows $J\left(f_{c}\right)$ admits no invariant line field as an added bonus.

Our own work addresses the infinitely renormalizable case. The main result of $[\mathrm{Mc}]$ is:

Theorem 5.2. If $f(z)=z^{2}+c$ is an infinitely renormalizable real quadratic polynomial, then $J(f)$ carries no invariant line field.

When combined with Yoccoz's result, this theorem implies a positive resolution to Conjecture NILF2 $\mathbb{R}$, which we restate as follows:

Corollary 5.3. Every component $U$ of the interior of the Mandelbrot set that meets the real axis is hyperbolic. 
In other words, if one runs the real axis through $M$, then all the fruit which is skewered is good.

Sketch of the proof of Theorem 5.2. By techniques of Sullivan [Sul3], the postcritical set $P(f)$ of an infinitely renormalizable real quadratic polynomial is a Cantor set with gaps of definite proportion at infinitely many scales. Using this information and abandoning the notion of a quadratic-like map, we construct instead infinitely many proper degree two maps $f^{n}: X_{n} \rightarrow Y_{n}$ (where we do not require that $X_{n} \subset Y_{n}$.) These maps range in a compact family up to rescaling. By the Lebesgue density theorem, any measurable line field $L_{z}$ looks nearly parallel on a small enough scale; using the dynamics, we transport this nearly parallel structure to $Y_{n}$ and pass to the limit. The result is a mapping with a critical point which nevertheless preserves a family of parallel lines, a contradiction. Thus the original map carries no invariant line field on its Julia set.

Remarks. In part, the structure of the argument parallels Sullivan's proof of Theorem 3.2; compactness of the mappings $f^{n}: X_{n} \rightarrow Y_{n}$ is a replacement for the finite-dimensionality of the group of Möbius transformations.

The proof also applies to certain complex quadratic polynomials, those which we call robust. For these maps, the notion of "definite gaps" in the postcritical Cantor set is replaced by a condition on the hyperbolic lengths of certain simple closed curves on the Riemann surface $\widehat{\mathbb{C}}-P(f)$.

Unfortunately, it is likely that robustness can fail for $z^{2}+c$ when $c$ is allowed to be complex. Counterexamples can probably be found using a construction of Douady and Hubbard, which also produces infinitely renormalizable quadratic polynomials whose Julia sets are not locally connected [Mil3].

\section{FURTHER DEVELOPMENTS}

To conclude, we mention three of the many other recent developments in complex dynamics which are most closely connected to the present discussion.

First, Swiagtek has announced a proof of Conjecture HD2 $\mathbb{R}$, the density of hyperbolic maps in the real quadratic family [Sw]. This result will settle the topological structure of bifurcations of real quadratic polynomials. Note that Conjecture HD2 $\mathbb{R}$ implies Theorem 5.2.

Second, Lyubich has announced a proof of the local connectivity of the Mandelbrot set at a large class of infinitely renormalizable points [Lyu2]. Thus it seems likely that Conjecture MLC itself is not too far out of reach. This conjecture, once settled, will complete our topological picture of the space of complex quadratic polynomials.

Finally, Shishikura has solved a long-standing problem about the geometry of the Mandelbrot set by proving that $\partial M$ (although it is topologically onedimensional) has Hausdorff dimension two [Shi]. To illustrate the complexity of the boundary of the Mandelbrot set, Figure 8 renders the image of $\partial M$ under the transformation $\log (z-c)$ for a certain $c \in \partial M .^{3}$ Note the cusp on the main cardioid in the upper right; looking to the left in the figure corresponds to zooming in towards the point $c$. (It is unknown at this time if $\partial M$ has positive

\footnotetext{
${ }^{3}$ Namely, $c=-0.39054087 \ldots-0.58678790 i \ldots$, the point on the boundary of the main cardioid corresponding to the golden mean Siegel disk.
} 


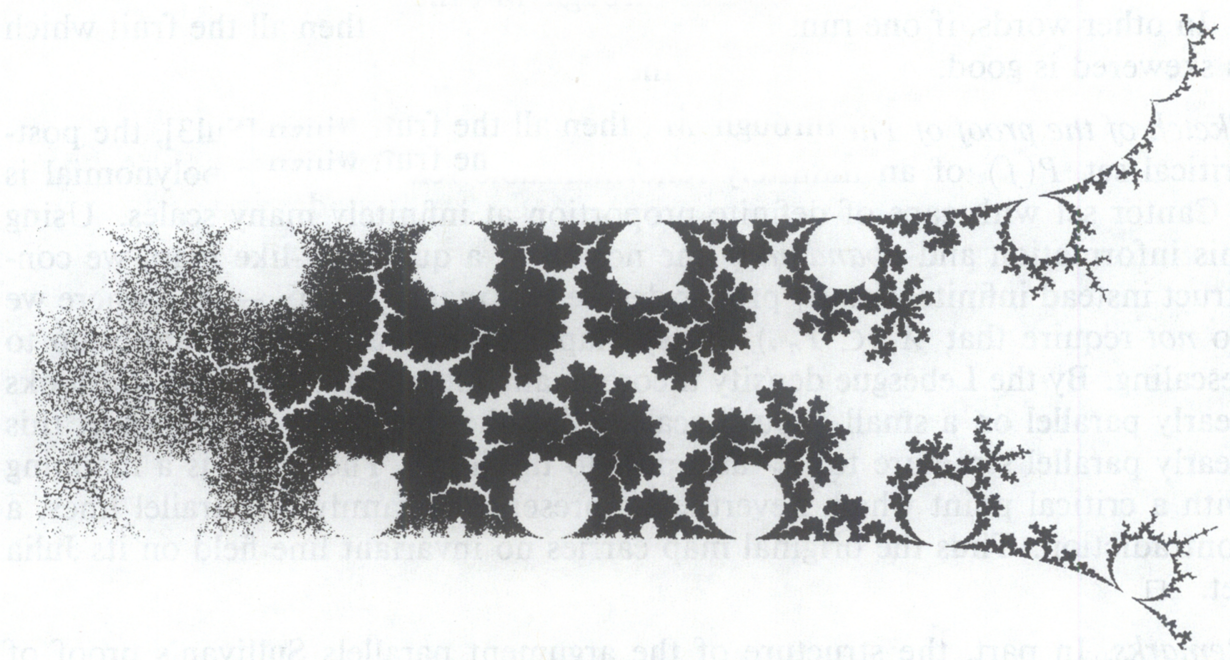

Figure 8. Log of the Mandelbrot set.

area; although the figure looks quite black in some regions, upon magnification these features resolve into fine filaments, apparently of area zero. Cf. [Mil1].)

In spite of these results and many others, the main conjectures in complex dynamics remain open. Our understanding of parameter space decreases precipitously beyond the setting of quadratic polynomials, and the realm of general rational maps contains much uncharted territory. For approaches to cubic polynomials and degree two rational maps, see [Mil4], [Mil5], [Rees2], [Rees3], [BH1], and [BH2].

\section{REFERENCES}

[Bea] A. Beardon, Iteration of rational functions, Springer-Verlag, New York, 1991.

[BR] L. Bers and H. L. Royden, Holomorphic families of injections, Acta Math. 157 (1986), 259-286.

[B1] P. Blanchard, Complex analytic dynamics on the Riemann sphere, Bull. Amer. Math. Soc. (N.S.) 11 (1984), 85-141.

[BH1] B. Branner and J. H. Hubbard, The iteration of cubic polynomials, Part I: The global topology of parameter space, Acta Math. 160 (1988), 143-206.

[BH2] - The iteration of cubic polynomials, Part II: Patterns and parapatterns, Acta Math. 169 (1992), 229-325.

[CG] L. Carleson and T. Gamelin, Complex dynamics, Springer-Verlag, New York, 1993.

[CS] S. S. Chern and S. Smale, eds., Global analysis, Amer. Math. Soc., Providence, RI, 1970.

[Cvi] P. Cvitanović, Universality in chaos, Adam Hilger Ltd., New York, 1984.

[Dou1] A. Douady, Systèmes dynamiques holomorphes, Astérisque 105-106 (1983), 39-64.

[Dou2] _ Descriptions of compact sets in C, Topological Methods in Modern Mathematics (L. R. Goldberg and A. V. Phillips, eds.), Publish or Perish, Cambridge, MA, 1993, pp. 429-465.

[DH] A. Douady and J. Hubbard, On the dynamics of polynomial-like mappings, Ann. Sci. École. Norm. Sup. (4) 18 (1985), 287-344.

[DMc] P. Doyle and C. McMullen, Solving the quintic by iteration, Acta Math. 163 (1989), 151180.

[EL] E. L. Eremenko and M. Lyubich, The dynamics of analytic transformations, Leningrad Math. J. 1 (1990), 563-634. 
[Fatou] P. Fatou, Sur les équations fonctionnelles (Deuxiéme mémoire), Bull. Sci. Math. France $\mathbf{4 8}$ (1920), 33-94.

[Hub] J. H. Hubbard, Local connectivity of Julia sets and bifurcation loci: Three theorems of J.-C. Yoccoz, Topological Methods in Modern Mathematics (L. R. Goldberg and A. V. Phillips, eds.), Publish or Perish, Cambridge, MA, 1993, pp. 467-511.

[Jak] M. V. Jakobson, Absolutely continuous invariant measures for one-parameter families of one-dimensional maps, Comm. Math. Phys. 81 (1981), 39-88.

[Lat] S. Lattès, Sur l'iteration des substitutions rationelles et les fonctions de Poincaré, C. R. Acad. Sci. Paris Sér. I Math. 166 (1918), 26-28.

[Lyu1] M. Lyubich, An analysis of the stability of the dynamics of rational functions, Selecta Math. Sov. 9 (1990), 69-90.

[Lyu2] _ Geometry of quadratic polynomials: Moduli, rigidity, and local connectivity, Stony Brook IMS Preprint 1993/9.

[Mane] R. Mañé, A proof of the $C^{1}$ stability conjecture, Publ. Math. Inst. Hautes Études Sci. 66 (1988), 160-210.

[MSS] R. Mañé, P. Sad, and D. Sullivan, On the dynamics of rational maps, Ann. Sci. École. Norm. Sup. (4) 16 (1983), 193-217.

[Mc] C. McMullen, Complex dynamics and renormalization, Princeton Univ. Press, Princeton, NJ (to appear).

[McS] C. McMullen and D. Sullivan, Quasiconformal homeomorphisms and dynamics III: The Teichmüller space of a holomorphic dynamical system, preprint.

[Mil1] J. Milnor, Self-similarity and hairiness in the Mandelbrot set, Computers in Geometry and Topology (M. C. Tangora, ed.), Lecture Notes in Pure and Appl. Math., Dekker, New York, 1989.

[Mil2] , Dynamics in one complex variable: Introductory lectures, Stony Brook IMS Preprint $1990 / 5$.

[Mil3] $\_$, Local connectivity of Julia sets: Expository lectures, Stony Brook IMS Preprint $1992 / 11$.

[Mil4] _ Remarks on iterated cubic maps, Experimental Math. 1 (1992), 5-24.

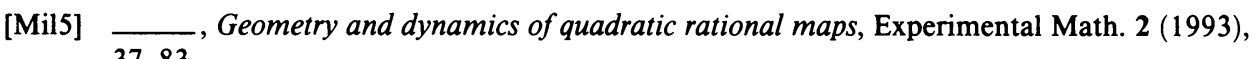
37-83.

[Rees1] M. Rees, Positive measure sets of ergodic rational maps, Ann. Sci. École. Norm. Sup. (4) 19 (1986), 383-407.

[Rees2] $\ldots$, Components of degree 2 hyperbolic rational maps, Invent. Math. 100 (1990), 357382.

[Rees3] __ A partial description of parameter space of rational maps of degree two: Part I, Acta Math. 168 (1992), 11-87.

[Shi] M. Shishikura, The Hausdorff dimension of the boundary of the Mandelbrot set and Julia sets, Ann. of Math. (to appear).

[Smale] S. Smale, Structurally stable systems are not dense, Amer. J. Math. 88 (1966), 491-496.

[Sul1] D. Sullivan, On the ergodic theory at infinity of an arbitrary discrete group of hyperbolic motions, Riemann Surfaces and Related Topics: Proceedings of the 1978 Stony Brook Conference, Ann. of Math. Stud., vol. 97, Princeton Univ. Press, Princeton, NJ, 1981.

[Sul2] - Conformal dynamical systems, Geometric Dynamics, Lecture Notes in Math., vol. 1007, Springer-Verlag, New York, 1983, pp. 725-752.

[Sul3] _ Bounds, quadratic differentials and renormalization conjectures, Mathematics into the Twenty-first Century: 1988 Centennial Symposium, August 8-12 (F. Browder, ed.), Amer. Math. Soc., Providence, RI, 1992.

[ST] D. Sullivan and W. P. Thurston, Extending holomorphic motions, Acta Math. 157 (1986), 243-258.

[Sw] G. Światek, Hyperbolicity is dense in the real quadratic family, Stony Brook IMS Preprint 1992/10. 
[Yoc1] J.-C. Yoccoz, Polynômes quadratiques et attracteur de Hénon, Astérisque 201-203 (1991), 143-165.

[Yoc2] __ Sur la connexité locale des ensembles de Julia et du lieu de connexité des polynômes, in preparation.

Mathematics Department, University of California, Berkeley, California 94720

E-mail address: ctm@math.berkeley.edu 